\begin{tabular}{ccc}
\hline & International Journal of Engineering \& Technology, $7(2.32)(2018) 57-59$ \\
SPC & Website: www.sciencepubco.com/index.php/IJET \\
Research paper & Jechnology \\
\hline
\end{tabular}

\title{
The Advanced Effectveness And Efficiency of New Testing Analysis
}

\author{
Dr.S.Srinivasa rao, G.Moulika, Lalitkumar .G, D. Naga Yeshwanth \\ K L E F, Green fields, Vaddeswaram, Guntur, Andhra Pradesh. 522502 \\ *Corresponding author E-mail: srinu1479cse@kluniversity.in
}

\begin{abstract}
Testing is the most widely used by the many software companies to develop the product or the project with most reliable. Testing will calculate the efficiency and effectiveness of the product to improve the performance of the project and work with proper way. Based on the testing results the product can be developed more effectively without showing any complications when the product is released. In this paper, the new integrated testing is developed to calculate the testing effectiveness and testing efficiency of the product at the time of releasing and at the time of development of the project. The existing system of this testing shows the early testing which is at the time of the development and the proposed system of the testing shows the late testing of the project i,e after the completion of the project. From the proposed system the cost, penalty, and benefit of a software during its development phase for finding the expected faults and predicted faults to release the product.
\end{abstract}

Keywords: Early testing, late testing, cost, penalty, benefit..

\section{Introduction}

Developing a huge software or websites or product based project is a most complicated issue. Exceptions and errors may come at every stage and be finding the bugs, errors is the tedious task for the programmers and also the testers. SDLC plays the major role in software testing. For every development of software project, it is mandatory to test the project to become the error-free project at the time of releasing. Developing the software is very costly. Every company according to their projects $50 \%$ of the cost is spending on testing. However, testing is the essential to ensure that the software is capable of doing what it was designed to do.

Testing is the costliest and it takes more time to complete the project development with testing. There two types of testing stages for every software project. Early testing and late testing. In this paper, the new testing techniques are implemented to analyze the testing results according to the stages such as design, coding, analysis, and maintenance. In the late testing it is not possible to know the analysis of the all the stages of the software project in late testing. There is the need for early testing also. The objective of the project is to reduce the testing time and improve the performance of the project. In this paper, the effectiveness and efficient of the project is calculated.

Testing is the step by step process to run the program and find the errors, exceptions, and bugs. It is also known that it is the process of checking and finalizing the software project or product based project which can check every page in the project and improve the performance of the project and check whether all the requirements are fulfilled or not [1]. The other meaning of testing is comparing the actual outcome and expected the outcome.

Adequacy and Efficiency assumes a vital part in programming testing. Test adequacy is the relative capacity of testing procedure to discover bugs in the product. Test productivity is the relative cost of finding a bug in the product under test. One of the significant issues in programming industry is testing time. Time implies cash and henceforth the whole procedure is expensive. Therefore to reduce testing cost it is necessary to reduce manual repeatedly testing time and number of test cases generated for particular system. Testing is a continuous process to check the software every time to make error free and it is impossible to make $100 \%$ error-free software. The STLC is the testing process to maintain and manage the whole process. In this paper, the syntactic dataset is used to show the results by calculating the late testing and early testing with the help of effectiveness and efficiency to improve the project.

\section{Related work}

Various valuable related estimations of testing thing level estimations are represented in the written work. George, Robert and Tammy [2] showed a course of action of estimations in the midst of the testing of NASA's MCCU. The usage of all the testing effort prompts recognize threats and issues in front of plan for the test method, restricted the impact of issues. The chiefs were given more information into the explanations behind issues, improving the suitability of response.

Rosenberg and Huffman [3] utilized computerized instrument to check necessities and their samples has check the route to utilization of new essentials for testing measures. Contraption is used to find out essentials that may be obligated to issues in the testing. In light of their examination work, they reason that estimations are available in the essentials stage to assess test plans. The undertakings to evaluate testing for the necessities.

Norman [4] worry about a bound together thing appraisal. His choice relied upon both judicious and survey use of steadfastness, peril and test estimations that is achievable to check and assess thing quality. 
Yanping Chen, Robert and Robenson [5] presented a honest to goodness undertaking coordinated by the IBM ECD test gathering to survey a course of action of estimations. They research the ampleness of a course of action of corresponding estimations for the parameters to evaluate idea of testing steps in perspective of the results of the errand. In future the consolidates appraisal of new estimations to check execution of individual test stages, offering recommendations to test gatherings and support bunches for essential modifications in the test method, and completing the full plan of estimations in an age test condition.

To find the bugs in the program the testing is the step by step process [1]. Supervises generally check the testing process by using programming specialist and test amass senses [6]. The researchers portrays testing as: "The route toward working a structure or section under decided conditions, watching or recording the result and making an appraisal of some piece of the system or fragment" and as "the path toward dismembering".

A famous physicist, Lord Kelvin said "when you can measure what you are discussing, and can express it in numbers, you know something about it; yet when you can't evaluate it, when you can't express it in numbers, your understanding is of melancholy and unacceptable kind; it may be the beginning of adapting, nonetheless you have scarcely in your examinations advanced to the period of science". Thus, unmistakably everything ought to be quantifiable. In the event that it isn't quantifiable, we should attempt to make it quantifiable [1].

Estimation lies at the core of numerous frameworks that represent our lives. It assumes a basic part on compelling programming advancement and gives the logical premise to programming testing. Estimation could be characterized as the procedure of exact, objective, task of numbers to properties of articles or occasions of this present reality so as to depict them [7]. It is the procedure by which numbers or images are appointed to qualities of substances in reality so as to portray them as per unmistakably characterized rules [8] Principal estimation is a methods by which numbers can be relegated by common laws to speak to the property, but does not surmise estimation of some other factors than the one being measured [9]. A compelling estimation action ought to have the capacity to assess the present procedure and give recommendation to the chief to future change [2].

\section{Programing Test Efficiency}

In every company the internal process of expanding is done with the available resources.

Every minute of time is important for the programmer to execute the project and to test the project.

At a certain point of view the test efficiency is measure for the code to check the code syntax.

$\mathrm{TE}=$ test efficiency;

$\mathrm{TP}=$ text productivity=no of bugs + migration + system $/$ add no of changes in program+ migration + system + user ack testing).

$\mathrm{TE}=$ no of changes resolved/total no of bugs identified) $* 100$.

The testing efficiency is calculating from the various equations utilized above.

PTE answer the changes of the test platform on the application.

Various calculations are utilized for STE (for various variables):$\mathrm{TE}=$ No of defects found divided by number of test cases executed .

- Test viability $=$ (add up to no of infused +total number of deformity found)/(add up to number of deformity escaped) $* 100$

- TE $=$ Loss because of issues/Total assets prepared by the framework.

\section{Existing system}

In this system, the synthetic dataset is taken from the various data sources and implemented the existing system. The effectiveness and efficiency of the existing system can be calculated based on the dataset. The project is divided into modules and every module have the developer, tester and manager to check the updates of the project For this late testing it is known that the processing is taken, the table-1 shows the processing details for existing system and table-2 show the result of the Existing System.

Table 1: Software Phase Data for Existing System Software Build Cycle Between2017-05-01 to 2017-10-11

\begin{tabular}{|l|l|}
\hline WorkLoad Scheduling Across SDLC Teams & \\
\hline \hline Analyzer's Size & 23 \\
\hline \hline Designer's Size & 16 \\
\hline \hline Developers's Size & 192 \\
\hline \hline Tester's Size & 80 \\
\hline Deployer's Size & 9 \\
\hline \hline Total's Size & 320 \\
\hline \hline Work Days Alloted & 164 \\
\hline \hline Work Days Scheduled & 163 \\
\hline
\end{tabular}

Table 2: Analysis using Late Testing in Existing System.

Software Upgradation CBP Defect Analysis Using Late Testing

\begin{tabular}{|c|l|l|l|}
\hline SDLC Stage & Initial & Phase & Total \\
\hline Analysis & 0 & 0 & 0 \\
\hline Design & 0 & 0 & 0 \\
\hline Coding & 3288 & 1494 & 4782 \\
\hline \hline Testing & 3372 & 1410 & 4782 \\
\hline
\end{tabular}

\section{Proposed method}

In the proposed system, the results shows the performance of the proposed system with synthetic dataset and table-4 shows the results for the propsed system with all the analysis of the dataset with the proposed system.

Table 3: Software Phase Data for Proposed System

Software Build Cycle Between2017-05-01 to 2017-10-11

\begin{tabular}{|l|l|}
\hline WorkLoad Scheduling Across SDLC Teams & \\
\hline Analyzer's Size & 27 \\
\hline \hline Designer's Size & 18 \\
\hline \hline Developers's Size & 223 \\
\hline \hline Tester's Size & 93 \\
\hline \hline Deployer's Size & 11 \\
\hline \hline Total's Size & 372 \\
\hline \hline Work Days Alloted & 164 \\
\hline Work Days Scheduled & 143 \\
\hline
\end{tabular}


Table 4: Software Up gradation with improved results for early testing.

Sotware Upgradation CBP Defect Analysis Using Early Testing

\begin{tabular}{|r|l|l|l|l|l|l|l|l|l|}
\hline SDLC Stage & Initial|(Previous) & Phase(Previous) & Initial|(Currenti) & Phase(Current) & Total & Benefit & Penalty & Cost \\
\hline \hline Analysis & 471 & 204 & 978 & 534 & 1512 & 0 & 2 & 54.0 \\
\hline \hline Design & 775 & 93 & 750 & 516 & 1266 & 1 & 1 & 10.0 \\
\hline Coding & 3996 & 1273 & 2892 & 1290 & 4182 & 1 & 1 & 10.0 \\
\hline \hline Testing & 5112 & 252 & 2898 & 1350 & 4248 & 1 & 1 & 10.0 \\
\hline Maintainence & 1 & 2201 & 1 & 2280 & 2281 & 1 & 1 & 0.0 \\
\hline \hline Total & & & & & 13480 & & & 84.0 \\
\hline
\end{tabular}

\section{Conclusion}

The proposed framework focus over the particular worries of the gathering of metric, for example, investigation, coding, outline, upkeep as a portion of the metrics are adequate to pick up in the pith of testing proficiency. Consequently, the outcomes show that precisely picked testing metric suite can be extremely valuable in programming item estimation and can give helpful data to test supervisors for basic leadership and future enhancements. The proposed framework gives a recorded view and reveals holes in existing exploration. The proposed framework depends on a uniform portrayal of new measurements and is helped by putting away the data in an organized archive for simplicity of handling.

\section{References}

[1] Myers, Glenford J., Corey Sandler, and Tom Badgett. The art of software testing. John Wiley \& Sons, 2011.

[2] George E. Stark, Robert C. Durst, Tammy M. Pelnik “An Evaluation of Software Testing metrics for NASA's Mission Control Center" 1992.

[3] Linda H. Rosenberg, Theodore F. Hammer, Lenore L. Huffman "Requirements, Testing, and Metrics" NASA GSFC, 1998.

[4] Norman F. Schneidewind "Measuring and Evaluating Maintenance Process Using Reliability, Risk, and Test Metrics" IEEE Transaction on Software Engineering, Vol. 25, No. 6, 1999.

[5] Yanping Chen, Robert L. Probert, Kyle Robenson "Effective Test Metrics for Test Strategy Evolution" Proceedings of the 2004 Conference of the centre for Advanced Studies on Collaborative Research CASCON'04.

[6] K. K. Aggarwal \& Yogesh Singh "Software Engineering Programs Documentation Operating Procedures (Second Edition)" New Age International Publishers, 2005.

[7] L. Finkelstein "Theory and Philosophy of Measurement", in Theoretical Fundamentals, Vol. 1, Handbook of measurement Science, P.H. Sydenlam, Ed. Chichester : John Wiley \& Sons, 1982, pp. 1 30.

[8] N. E. Fenton and S. L. Pfleager "Software Metrics: A Rigorous and Practical Approach", Second Edition Revised ed. Boston: PWS Publishing, 1997.

[9] W. Torgerson, S., "Theory and Methods of Scaling" New York: John Wiley \& Sons, 1958. 